\title{
Reproducción de Micropterus salmoides (Pisces: Centrarchidae), en el embalse Gustavo Díaz Ordaz, Sinaloa, México
}

\author{
Rigoberto Beltrán Alvarez ${ }^{1}$, Jesús Sánchez Palacios ${ }^{1}$, Juan Pedro Ramírez Lozano ${ }^{1}$ \\ \& Adolfo-Armando Ortega Salas ${ }^{2}$ \\ 1. Laboratorio de Limnología y Pesquerías de Agua Dulce. Facultad de Ciencias del Mar. Universidad Autónoma de \\ Sinaloa. Paseo Claussen s/n, Col. Los Pinos, Mazatlán, Sin. México; rigober80@hotmail.com, jsp45@hotmail.com, \\ juanpedro16@live.com.mx \\ 2. Instituto de Ciencias del Mar y Limnología. Universidad Nacional Autónoma de México. Av. Joel Montes Camarena \\ S/N Apartado Postal 811 C.P. 82040, Mazatlán, Sin. México; ortosal@cmarl.unam.mx
}

Recibido 22-VI-2012. Corregido 10-XII-2012. Aceptado 22-I-2013.

\begin{abstract}
Micropterus salmoides (Pisces: Centrarchidae) reproduction in the Gustavo Díaz Ordaz reservoir, Sinaloa, México. Micropterus salmoides is an important fish species for sport fishing activities, condition that has promoted its introduction to different reservoirs in Mexico and worldwide. With the aim to improve its fisheries management, this research dealt with some reproductive aspects of this species in the Gustavo Díaz Ordaz reservoir, where it was studied from August 2008 through March 2011. To this end, we obtained 938 specimens, with gillnets of different sizes, to determine their total length ( $\mathrm{Lt}$, in $\mathrm{cm})$, weight ( $\mathrm{Pt}$, in $\mathrm{g})$, sex, gonadosomatic index, condition factor, fecundity and size at first maturity. Lt and Pt ranged from 15.9 to $63 \mathrm{~cm}$ (37.4 \pm 78.0$)$ and 57 to $4431 \mathrm{~g}(731.7 \pm 619.0)$, respectively. The Pt-Lt relationship showed a positive allometric growth, with no significant difference between males and females $(\mathrm{F}=0.9955, \mathrm{p}=0.3187)$. The male: female ratio obtained was 1:0.83. Mass spawning lasted from December to April. Size at first maturity was $33.7 \mathrm{~cm}$ and average fecundity was $32294 \pm 12878.7$ oocytes/female. The gonadosomatic index was low from May through November, and increased between January and March. The condition factor was high before the spawning season and decreased after the reproductive period. We recommend a fishing ban from January to March, and to allow the capture size between 33 and 40cm. Rev. Biol. Trop. 61 (3): 1313-1325. Epub 2013 September 01.
\end{abstract}

Key words: reproduction, Micropterus salmoides, fisheries management, reservoir.

La lobina negra Micropterus salmoides (Lacépède 1802) es una especie cuya distribución natural se extiende desde la parte oriental de Canadá hasta la cuenca del río Bravo, en el noroeste de México. Sin embargo, se ha propagado artificialmente de manera considerable, por lo que actualmente se encuentra en numerosas localidades donde antes no existía (Carlander 1977, Page \& Burr 1991). Fue traída a México procedente de los Estados Unidos Americanos en 1901, los primeros especímenes fueron introducidos en la presa La Boquilla, Chihuahua, conocida como Lago Conchos (Robbins \& MacGrimmon 1974), y se ha diseminado en la mayoría de los cuerpos dulceacuícolas de México cumpliendo con el objetivo de su introducción, al pasar a formar parte de las especies explotadas comercialmente, $\mathrm{y}$ al favorecer el desarrollo de actividades pesqueras y turísticas. En Latinoamérica, su presencia se reporta en Brasil donde llegó desde 1922 (Schutz \& Leal 2005), en Portugal desde 1952 (Godinho \& Ferreira 1996), y se informa de su presencia en la región de la península de Iberia (Coop et al. 2004) donde se le encuentra en la mayoría de los ríos y lagos Españoles (Nicola et al. 1996). En Zimbabwe (Beamish et al. 2005), señalan que llegó en 1932. El objetivo de su introducción en países de regiones tropicales, subtropicales y 
templados, ha sido el de generar una actividad pesquera y turística en base a la pesca deportiva de esta especie.

Por considerarse una especie valiosa desde el punto de vista nutricional, es un alimento muy preciado por lo cual alcanza una importante cotización en el mercado, situación por la cual en algunos cuerpos de agua es sujeta a una intensa depredación mermando la abundancia de sus poblaciones.

En México, los reportes de su captura en actividades pesqueras apenas rebasan las 1 300ton (Anuario Estadístico de Pesca 2009), su producción ha sido menor al de otras especies piscícolas de agua dulce, lo que se debe a que en la mayoría de los embalses esta especie se encuentra reservada a actividades de pesca deportiva y los datos existentes provienen de reportes de pesca incidental.

Entre los aspectos biológicos de la lobina que han sido estudiados se encuentra la edad y el crecimiento (Guzmán-Arroyo et al. 1978, Anderson 1980, Medina et al.1994, Pérez 2000, Schulz \& Leal 2005, Rodríguez-Sánchez et al. 2009), y la alimentación (Beltrán et al. 2004, Beamish et al. 2005, Castañeda \& Silva 2005).

El estudio del ciclo reproductivo de las especies que conforman los recursos pesqueros explotados es de vital importancia, dado que su conocimiento permite recomendar las épocas en que la especie se debe proteger mediante veda reproductiva, para evitar su extracción cuando los organismos se están reproduciendo (Rodríguez 1992, Saborido 2005).

En el presente estudio se presenta información sobre aspectos reproductivos de la lobina negra $M$. salmoides en la presa Gustavo Díaz Ordaz, con el propósito de ampliar el conocimiento sobre su biología y contribuir a una administración adecuada del recurso.

\section{MATERIALES Y MÉTODOS}

La presa Gustavo Díaz Ordaz es regionalmente conocida como "Bacurato", fue construida en el año de 1982, sobre el cauce del río Sinaloa, en el municipio de Sinaloa de Leyva, a $56 \mathrm{~km}$ al norte de la ciudad de Guasave; geográficamente, se localiza sobre los $25^{\circ} 54^{\prime}$ N - $107^{\circ} 54^{\prime}$ W, en el estado de Sinaloa, México.

Se realizaron recolectas bimensuales de organismos de agosto 2008 a marzo 2011, para lo cual se utilizaron dos juegos redes agalleras de monofilamento de $0.30 \mathrm{~mm}$ de diferentes luz de malla ( 3 ", 3 11/2,"3 3/4", ,4", 4 1/2", 4 3/4" 4 7/8", 5 "). Las artes de pesca se colocaron al atardecer en diferentes puntos del embalse, y por la mañana del día siguiente, los organismos se recogían y se separaban por tipo de red, procediendo a tomar las siguientes mediciones: longitud total $(\mathrm{Lt})$, longitud patrón $(\mathrm{Lp})$ y altura $(\mathrm{H})$, para lo que se utilizó un ictiómetro convencional $( \pm 1 \mathrm{~mm})$. El peso total $(\mathrm{Pt})$ y eviscerado $(\mathrm{Pe})$ se obtuvieron con una balanza digital Ohaus con capacidad de $10000 \mathrm{~g}( \pm 1 \mathrm{~g})$. Para conocer la estructura poblacional se realizaron cálculos de parámetros básicos de estadística así como los histogramas de longitud y peso por sexos. La relación longitud-peso se obtuvo con el modelo potencial, cuya expresión matemática de acuerdo a Ricker (1975) se define por: $\mathrm{P}_{\mathrm{t}}=\mathrm{a}$ $(\mathrm{Lt})^{\mathrm{b}}$ donde: $\mathrm{P}_{\mathrm{t}}=\mathrm{el}$ peso total, $\mathrm{L}_{\mathrm{t}}=$ longitud total, $a=$ intercepto y $b=$ exponente también llamado coeficiente de alometría.

Para establecer el sexo de manera visual, se disectaron los organismos exponiendo sus órganos sexuales anotando si pertenecía a macho (M), hembra $(\mathrm{H})$ o indiferenciado (I). Se estableció la proporción sexual para cada muestreo y la diferencia entre la relación teórica 1:1 y la encontrada se estableció mediante la prueba "Ji cuadrada" (Zar 1984). El grado de desarrollo de sus gónadas se estableció de acuerdo a la escala universal de seis fases de madurez para reproductores totales de Kesteven (1975). Sin embargo, debido a la confusión que causó la separación de las primeras fases de maduración, así como estadio VI indicador de organismos desovados, solo se registraron los estadios claramente diferenciables (II, III y IV). La fecundidad absoluta se estableció a partir del conteo de ovocitos mediante el método gravimétrico según la relación:

$\mathrm{F}=\mathrm{nG} / \mathrm{g}$ donde: $\mathrm{F}=$ Fecundidad, $\mathrm{n}=$ Número de ovocitos en la submuestras 
$\mathrm{G}=$ peso de ambas gónadas y $\mathrm{g}=$ peso de la submuestras.

El índice gonadosomático se calculó con la relación: $\mathrm{IG}=\mathrm{P}_{\mathrm{g}} / \mathrm{P}_{\mathrm{t}}(100)$ donde:

$I G=$ =Índice gonadosomático, $\mathrm{P}_{\mathrm{g}}=$ Pesode ambas gónadas, $\mathrm{P}_{\mathrm{t}}=$ Peso total del pez (Rosenblum et al. 1987).

El factor de condición de Fulton (Bagenal 1978), se calculó para machos y hembras mediante la relación: $\mathrm{K}=\mathrm{P}_{\mathrm{t}} / \mathrm{L}^{\mathrm{b}}\left(10^{5}\right)$ donde: $K=$ factor de condición

$\mathrm{P}_{\mathrm{t}}=$ Peso total, $\mathrm{L}_{\mathrm{t}}=$ Longitud total, $b=$ coeficiente de alometría de la relación longitud peso $\mathrm{y}$, $105=$ factor de ajuste a la unidad.

La talla de primera madurez fue estimada a través del modelo logístico:

$\mathrm{P}=1 / 1+\mathrm{e}^{(\mathrm{a}-\mathrm{bLt})}$ donde: $P=\mathrm{es}$ la proporción de individuos maduros, $a$ y $b=$ parámetros del modelo. Posteriormente, la talla promedio de primera madurez (Lm50\%) fue estimada por medio de: $\mathrm{Lm}_{50}=\mathrm{a} / \mathrm{b}$ (Sparre \& Venema 1997).

\section{RESULTADOS}

Estructura de la población: En total se recolectaron 938 organismos de M. salmoides. $\mathrm{Al}$ ordenar los datos en frecuencias de longitud de los peces, se observó que la longitud mínima obtenida para las hembras fue de $15.9 \mathrm{~cm}$ y la máxima de $63 \mathrm{~cm}(35.2 \pm 8.23)$, y para los machos una mínima de 19.9 y una máxima de 45.6 (32.2 \pm 4.21$)$. El peso más bajo registrado en hembras fue de $57 \mathrm{~g}$ y el más alto fue de 4

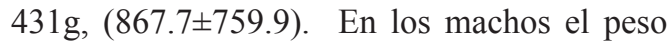
varió entre $90 \mathrm{~g}$ como mínimo y $1424 \mathrm{~g}$ como máximo; (571.6 \pm 241.2$)$ (Cuadro 1 y Fig. 1). En los histogramas se observa que la mayor parte de la población evaluada fluctúo de los 27 a $41 \mathrm{~cm}$ y entre los 250 a $1450 \mathrm{~g}$.

Relación longitud total peso total: $\mathrm{Al}$ relacionar las variables longitud total contra peso total, se observó que se ajustan a un modelo tipo potencial, el cual para las hembras fue $\mathrm{P}_{\mathrm{t}}=0.011(\mathrm{Lt})^{3.106}$ con un coeficiente de correlación $\mathrm{r}=0.9864(\mathrm{p}<0.001)$ y para los machos fue $\mathrm{P}_{\mathrm{t}}=0.009(\mathrm{Lt})^{3.151}$ con un coeficiente de correlación $\mathrm{r}=0.9679(\mathrm{p}<0.001)$; al aplicar la prueba de Ancova se comprobó que no existe diferencia entre hembras y machos $(\mathrm{F}=0.9955$, $\mathrm{p}=0.3187$ ). Para establecer el tipo de crecimiento de los organismos se aplicó la prueba estadística de " $t$ "student al exponente (b) de las relaciones longitud-peso de cada sexo, de lo cual resultó un valor de $\mathrm{b}$ significativamente mayor que 3 , por lo que se consideró el crecimiento como alométrico positivo tanto en hembras $(\mathrm{t}=4.2077, \mathrm{p}<0.05)$ como en machos $(\mathrm{t}=4.1288, \mathrm{p}<0.05)$ (Fig. 2).

Proporción de sexos: Del total de los organismos que fueron sexados durante el ciclo de estudio, 410 fueron hembras, 495 machos y 33 indiferenciados. La proporción sexual macho-hembra (M:H) global fue de 1:0.83. Se observó que los machos dominaron en 10

CUADRO 1 / TABLE 1

Estadísticos descriptivos de la longitud y peso / Descriptive statistics of the length and weight

\begin{tabular}{lcccc} 
& \multicolumn{2}{c}{ Longitud total } & \multicolumn{2}{c}{ Peso total } \\
& Hembras & Machos & Hembras & Machos \\
Mínimo & 15.9 & 19.9 & 57 & 90 \\
Máximo & 63 & 45.6 & 4431 & 1424 \\
Desviación estándar & 8.2347 & 4.21 .15 & 759.9 & 241.2 \\
Varianza & 67.8 & 17.7 & 577467 & 58173 \\
Media & 35.2 & 32.2 & 867.76 & 571.69 \\
Moda & 33 & 31 & 616 & 374 \\
\hline
\end{tabular}




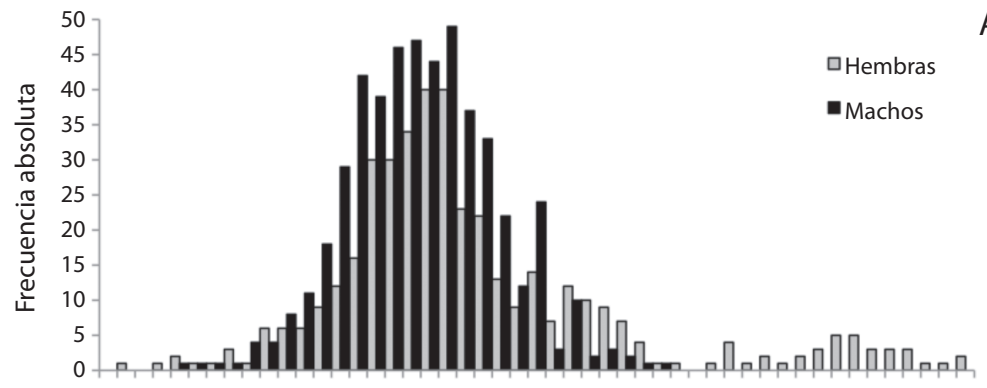

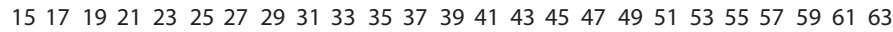
Longitud total $(\mathrm{cm})$

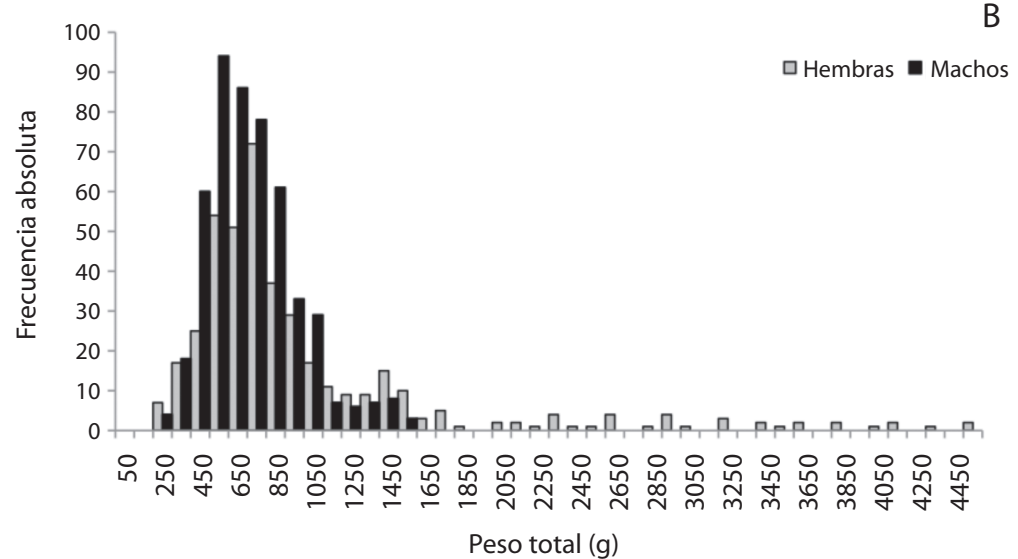

Fig. 1. Frecuencia absoluta de Micropterus salmoides. Longitud total (A), peso total (B). Fig. 1. Absolute frequency of Micropterus salmoides. Total length (A), weight (B).

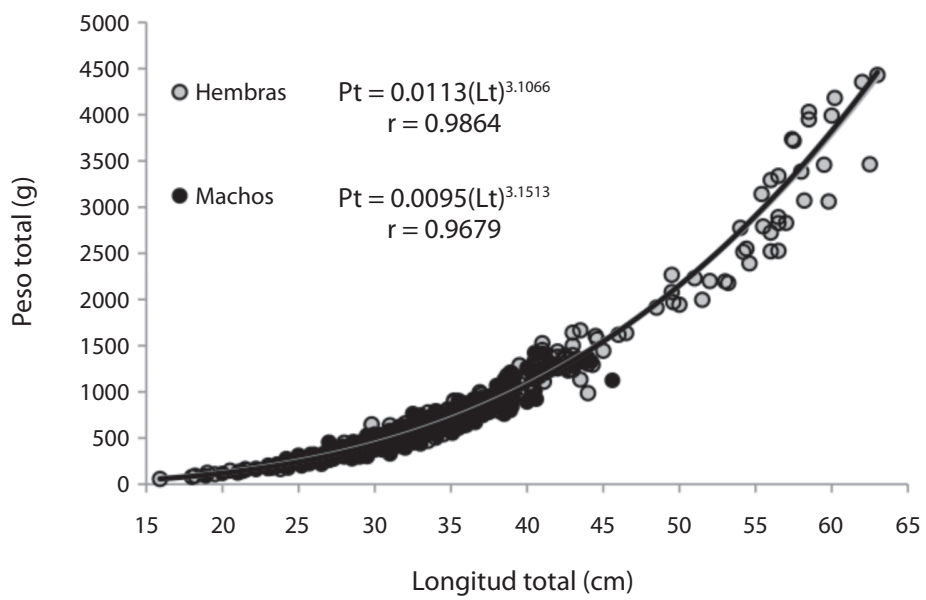

Fig. 2. Relación longitud total-peso total. / Fig. 2. Length-weight relationship. 
de los 20 muestreos realizados, las hembras dominaron en los meses restantes. La mayor diferencia entre los sexos se presentó en septiembre, al obtenerse 29 hembras y un solo macho $(1 \mathrm{H}: 0.034 \mathrm{M})$; en contra parte, la proporción sexual se igualó a 14 en junio y a 25 en octubre 2009. Los organismos para los que no fue posible identificar su sexo, fueron observados mayormente en agosto 2008 (Cuadro 2).

Madurez gonadal: Basándose en los datos de madurez gonadal obtenidos durante el ciclo de muestreo, se encontraron organismos en estadio II (inmaduros) en la mayoría de los meses de muestreo, pero fueron más abundantes de mayo a noviembre. En cuanto al estadio III (en maduración), aparecieron en mayor porcentaje de septiembre a diciembre 2008, diciembre 2009; junio y noviembre 2010, mientras que los peces con gónadas en máxima maduración y desovados, estuvieron presentes de diciembre a abril en los diferentes ciclos de muestreo (Fig. 3). Lo anterior evidencia que el proceso de maduración de las gónadas de $M$. salmoides en la presa Gustavo Díaz Ordaz, se inicia en septiembre con desoves desde diciembre, los cuales se extienden hasta abril.

Índice gonadosomático: En la figura $4 \mathrm{se}$ muestran los promedios mensuales y la evolución del índice gonadosomático desde agosto 2008 hasta marzo 2011. En agosto, septiembre y octubre de cada año se presentaron los valores más bajos de este índice, lo cual es un indicador de que los especímenes de $M$. salmoides se encuentran en la etapa de descanso reproductivo; en diciembre se registraron los especímenes con sus gónadas en la primera etapa de maduración, lo cual se refleja en que el índice gonadosomático empieza a incrementarse y para enero-marzo se registran los valores más altos, coincidiendo con la presencia de gónadas en estadios de máxima maduración. Aunque en menores proporciones, los organismos maduros se registraron hasta mayo.

CUADRO 2 / TABLE 2

Proporción sexual / Sexual proportion

\begin{tabular}{lcccccccl} 
Mes & Hembras & Machos & Indif & Total & M:H & $X^{2}$ & Probabilidad & \multicolumn{1}{c}{ Significancia } \\
Ago-08 & 7 & 3 & 24 & 27 & $1: 0.4$ & 1.7000 & 0.1923 & no significativa \\
Sep-08 & 29 & 1 & & 30 & $1: 0.0$ & 26.1667 & 0 & significativa \\
Oct-08 & 26 & 10 & & 36 & $1: 0.4$ & 7.1389 & 0.0075 & significativa \\
Dic-08 & 16 & 10 & 5 & 31 & $1: 0.6$ & 1.4231 & 0.2329 & no significativa \\
Ene-09 & 20 & 37 & & 57 & $1: 1.9$ & 5.0877 & 0.0241 & significativa \\
Feb-09 & 9 & 6 & & 15 & $1: 0.7$ & 0.6667 & 0.4142 & no significativa \\
Mar-09 & 24 & 51 & & 75 & $1: 2.1$ & 9.7333 & 0.0018 & significativa \\
May-09 & 10 & 9 & 4 & 23 & $1: 0.9$ & 0.1053 & 0.7456 & no significativa \\
Jun-09 & 14 & 14 & & 29 & $1: 1.0$ & 0.0357 & 0.8501 & no significativa \\
Ago-09 & 39 & 54 & & 93 & $1: 1.4$ & 2.4301 & 0.119 & no significativa \\
Oct-09 & 25 & 25 & & 50 & $1: 1.0$ & 0.02 & 0.8875 & no significativa \\
Dic-09 & 28 & 45 & & 73 & $1: 1.6$ & 3.9726 & 0.0462 & significativa \\
Ene-10 & 27 & 28 & & 55 & $1: 1.0$ & 0.0364 & 0.8488 & no significativa \\
Mar-10 & 12 & 9 & & 21 & $1: 0.8$ & 0.4762 & 0.4902 & significativa \\
Abr-10 & 48 & 54 & & 102 & $1: 1.1$ & 0.3627 & 0.547 & no significativa \\
Jun-10 & 6 & 39 & & 45 & $1: 6.5$ & 24.2222 & 0 & significativa \\
Jul-10 & 1 & 19 & & 20 & $1: 19.0$ & 16.2502 & 0.001 & significativa \\
Nov-10 & 29 & 39 & & 68 & $1: 1.3$ & 1.4853 & 0.2229 & no significativa \\
Ene-11 & 20 & 24 & & 44 & $1: 1.2$ & 0.3864 & 0.5342 & no significativa \\
Mar-11 & 20 & 18 & & 38 & $1: 0.9$ & 0.1316 & 0.7168 & no significativa \\
Total & 410 & 495 & 33 & 938 & $1: 1.2$ & & &
\end{tabular}




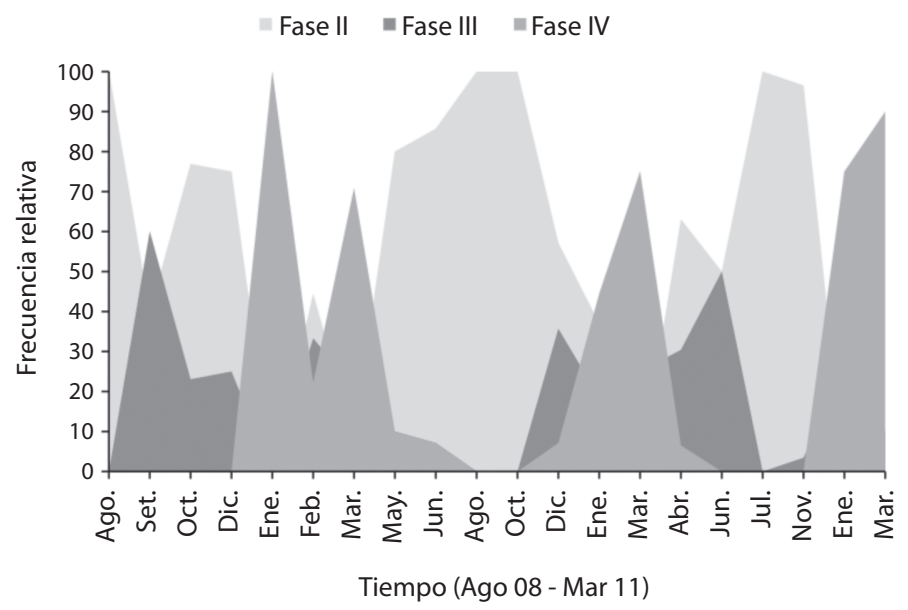

Fig. 3. Madurez gonadal. / Fig. 3. Gonadal maturity.

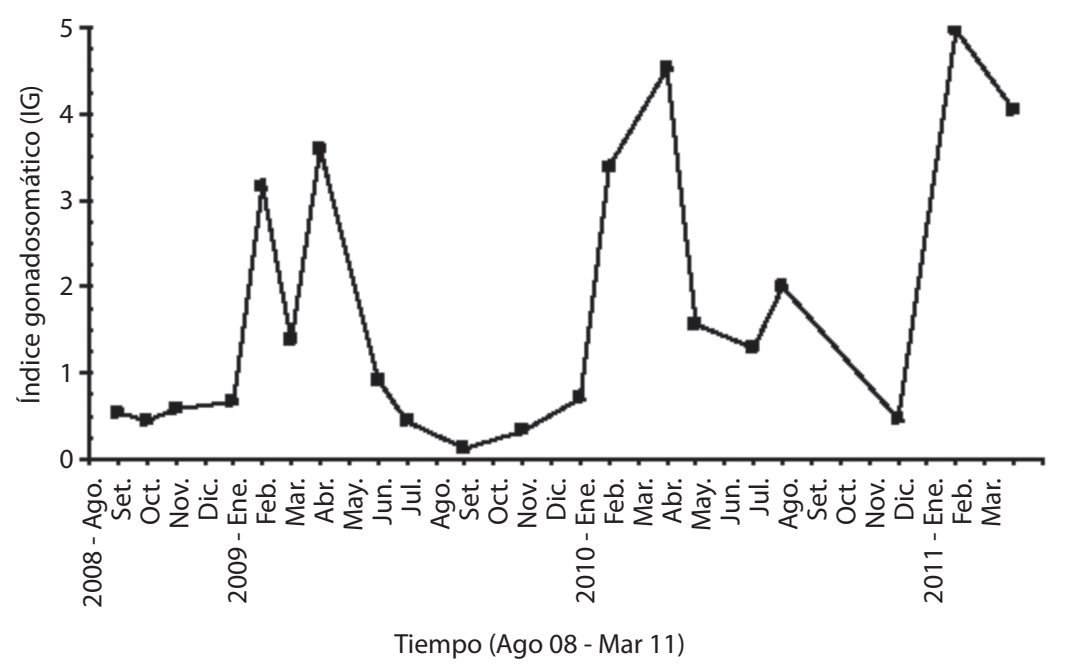

Fig. 4. Índice gonadosomático en hembras. / Fig. 4. Gonadosomatic index in females.

Factor de condición: El factor de condición en organismos hembras de $M$. salmoides registró un incremento de octubre 2008 a julio 2010, con los valores máximos en enero y octubre 2009. Los valores mínimos se observaron en agosto y septiembre 2008, noviembre 2010, enero y marzo 2011 (Fig. 5a). En los machos, se observaron los valores más altos desde octubre 2008 a noviembre 2010, exhibiendo su valor máximo en diciembre 2009 y los mínimos en agosto 2008, enero y marzo 2011 (Fig. 5b).
Fecundidad: El número de ovocitos por intervalo de tallas se presenta en el cuadro 3. Se observa que el promedio más bajo fue de $9537.3 \pm 2681.2$ ovocitos para un intervalo de talla de $28.5-29.5 \mathrm{~cm}$ y $310-497 \mathrm{~g}$, en tanto que el más alto fue de $37092 \pm 14324$ ovocitos correspondiente al intervalo de longitud de 44.6-57.5 y $1293-3796 \mathrm{~g}$ de peso.

Al relacionar la fecundidad contra la longitud total $(\mathrm{cm})$ y peso total $(\mathrm{g})$ se observó que se ajustan a un modelo de tipo exponencial 

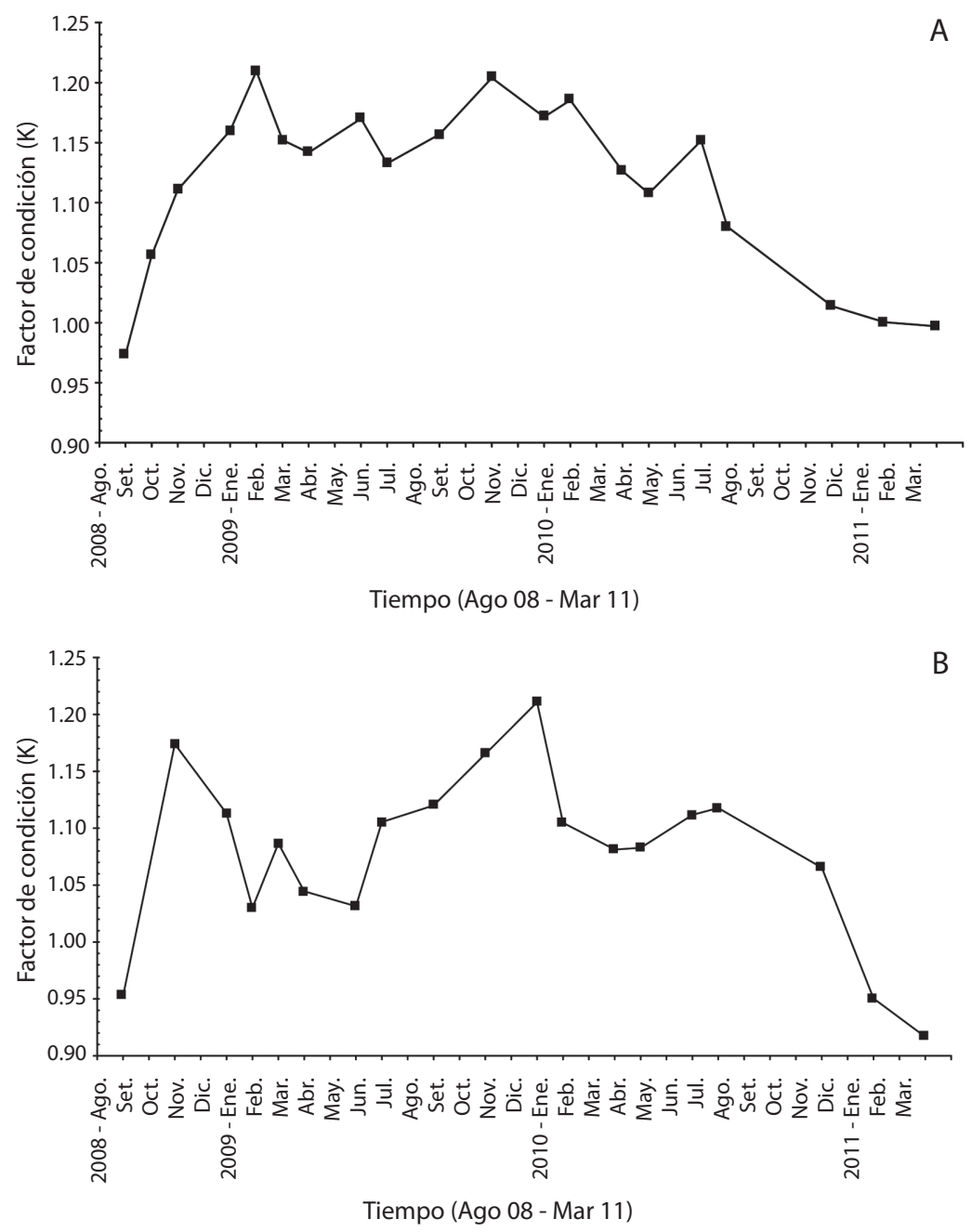

Fig. 5. Factor de condición en hembras y machos. / Fig. 5. Condition factor in females and males.

\section{CUADRO 3 / TABLE 3}

Fecundidad con respecto a la longitud y peso total / Fecundity with respect to length and total weight

\begin{tabular}{ccccc} 
Long total $(\mathrm{cm})$ & Peso total $(\mathrm{gr})$ & Intervalo de $(\mathrm{MG})$ & Media \pm D.E & Promedio ovocitos/cm \\
$28.6-29.5$ & $310-497$ & $3-5$ & $9537.3 \pm 2681.2$ & 26.1 \\
$29.6-30.5$ & $387-539$ & $3-5$ & $10853.8 \pm 3091.6$ & 22.9 \\
$30.6-31.5$ & $448-626$ & $3-5$ & $14734.3 \pm 7006.2$ & 28.6 \\
$31.6-32.5$ & $472-550$ & $4-5$ & $10019.4 \pm 1488.3$ & 19.8 \\
$32.6-33.5$ & $470-611$ & $4-5$ & $14322.4 \pm 3196.2$ & 27.2 \\
$33.6-34.5$ & $504-605$ & $4-5$ & $12060.5 \pm 5735.1$ & 19.4 \\
$34.6-35.5$ & $541-746$ & $4-5$ & $2023.1 \pm 8493.8$ & 29.6 \\
$35.6-36.5$ & $575-786$ & $4-4$ & $15060.8 \pm 8753.0$ & 21.1 \\
$36.6-37.5$ & $611-879$ & $4-5$ & $15622.8 \pm 58619$ & 26.1 \\
$37.6-38.5$ & $642-979$ & $3-5$ & $15531.8 \pm 7452.2$ & 16.9 \\
\hline
\end{tabular}


CUADRO 3 (Continuación) / TABLE 3 (Continued)

Fecundidad con respecto a la longitud y peso total / Fecundity with respect to length and total weight

\begin{tabular}{ccccc} 
Long total $(\mathrm{cm})$ & Peso total $(\mathrm{gr})$ & Intervalo de $(\mathrm{MG})$ & Media \pm D.E & Promedio ovocitos/cm \\
\hline $38.6-39.5$ & $937-1181$ & $3-5$ & $19562.8 \pm 9066.0$ & 21.7 \\
$39.6-40.5$ & $985-1275$ & $3-5$ & $26683.4 \pm 11544.6$ & 23.6 \\
$40.6-41.5$ & $1134-1527$ & $4-5$ & $26854.4 \pm 6322.5$ & 22.2 \\
$41.6-42.5$ & $1208-1437$ & $4-5$ & $2476.3 \pm 10244.0$ & 20.6 \\
$42.6-43.5$ & $1321-1641$ & $4-5$ & $32933.0 \pm 14544.8$ & 22.1 \\
$43.6-57.5$ & $1293-3796$ & $4-5$ & $37092.2 \pm 14324.8$ & 25.1 \\
\hline
\end{tabular}

como se muestra en la figura 6; Fecundidad $\mathrm{L}_{\mathrm{t}}=F=745.58 \mathrm{e}^{0.0846(\mathrm{Lt})}$ con un coeficiente de correlación $\mathrm{r}=0.6605$ y Fecundidad: $\mathrm{P}_{\mathrm{t}}=F=6618.5 \mathrm{e}^{(\mathrm{PT})}$ con un coeficiente de correlación $\mathrm{r}=0.7115$.
Talla de primera madurez: La talla de primera madurez en hembras se calculó con la proporción de individuos maduros contra las tallas correspondientes, por lo que la talla promedio de primera madurez fue de $33.7 \mathrm{~cm}$ (Fig. 7).
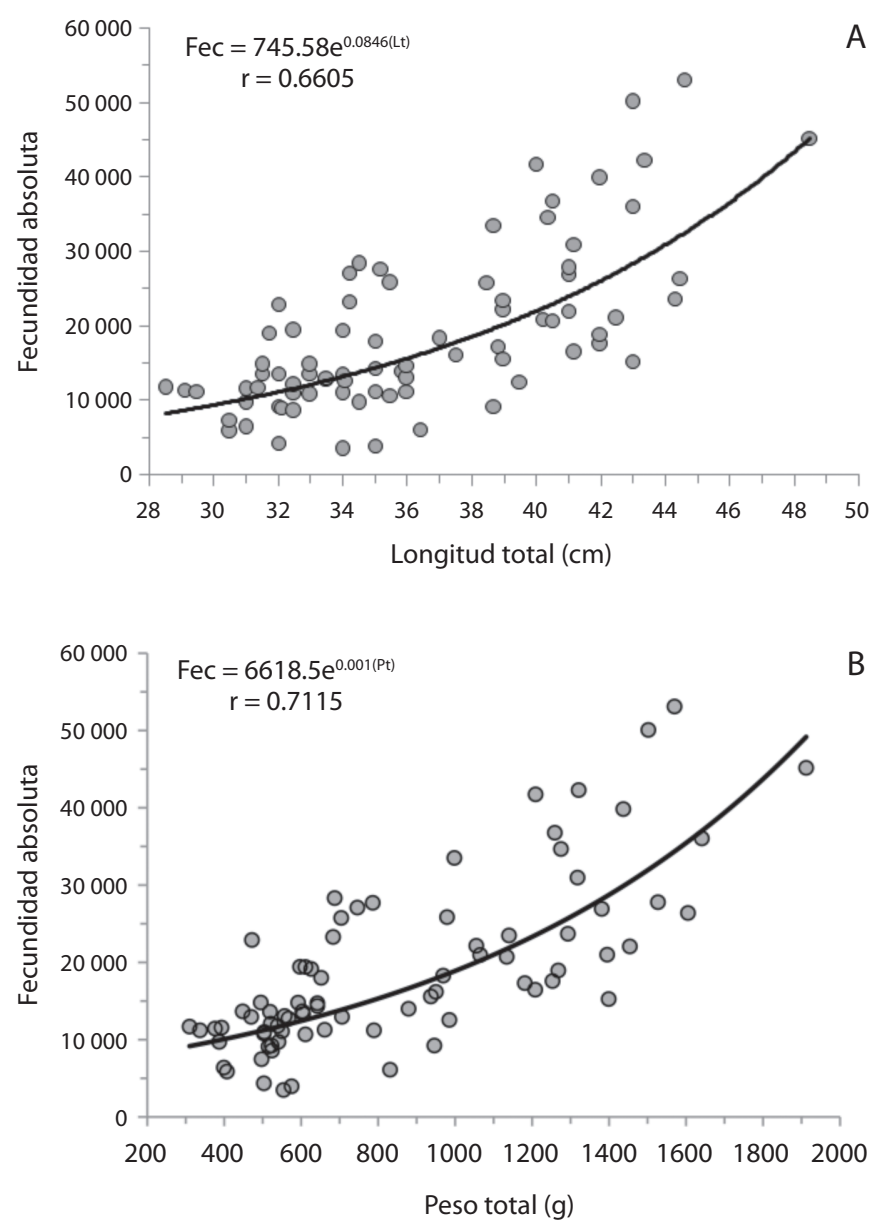

Fig. 6. Relación fecundidad longitud total (A) y peso total (B). / Fig. 6. Fecundity and total length and weight relationship. 


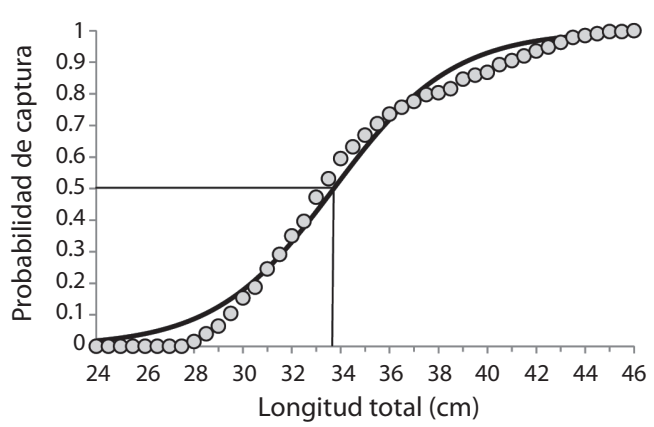

Fig. 7. Talla de primera madurez en hembras.

Fig. 7. Size of first maturity in females.

\section{DISCUSIÓN}

La amplitud en la talla y peso encontrada en la estructura poblacional de $M$. salmoides se debió a que en su captura se utilizaron redes agalleras de 3 a 5 pulgadas de luz de malla, por ello los histogramas de frecuencias absolutas, tanto de longitud como de peso, presentaron una distribución asimétrica hacia la derecha, por lo cual, la media resultó ligeramente mayor que la moda. Los especímenes mayores de $45 \mathrm{~cm}$ y $1650 \mathrm{~g}$ de peso total fueron todas hembras. Anderson (1980) señala que en pesca deportiva a los especímenes de estas tallas, se les clasifica dentro de la categoría de preferente $\mathrm{y}$ trofeo, y son los que atraen al turismo nacional y extranjero que gustan de esta actividad.

La estructura de tallas y pesos de lobina que reporta Pérez (2000) para la presa El Salto, Sinaloa, México fue de 7.7 a $59.9 \mathrm{~cm}$ y pesos de $104 \mathrm{~g}$, datos que resultan similares a los encontrados en este estudio; en esto quizás tiene que ver la política de pesca implementada por los prestadores de servicios de pesca deportiva junto con autoridades de pesca, que consiste en capturar y soltar las lobinas, lo cual probablemente ha contribuido tanto el incremento de la población como al aumento de tallas y pesos. Olague (2007), en la presa José López Portillo, Sinaloa, México, observó una longitud máxima de $59 \mathrm{~cm}$ y la mínima de $20.2 \mathrm{~cm}$, con un promedio $31.7 \mathrm{~cm}$ y desviación estándar de $5.6 \mathrm{~cm}$.

La relación longitud-peso brinda información de la proporción en que crecen estas dimensiones de los peces, si su pendiente es igual a tres, los peces tendrán un crecimiento de tipo isométrico y si este es menor o mayor a tres, será alométrico (Riker 1975). El análisis estadístico de las pendientes (3.106 en hembras y 3.151 en machos) permitió establecer que en ambos sexos la lobina registró un crecimiento de tipo alométrico positivo, lo que significa que cambios en la forma, talla y peso no son constantes a través del tiempo (Riker 1975). Roque (1992) reporta que la lobina en la presa Vicente Guerrero, Tamaulipas registra un tipo de crecimiento alométrico. Olague (2007) reporta un valor de la pendiente de la relación longitudpeso de 3.413 , el cual resultó estadísticamente diferente de tres por lo que el crecimiento de esta especie en la presa José López Portillo fue de tipo alométrico positivo; en este caso, los cambios en el cuerpo del pez no son constantes, lo que se puede deber a los cambios en las condiciones ambientales, su calidad de depredador, disponibilidad de alimento, entre otros.

La proporción global entre machos y hembras mostró una ligera dominancia de los primeros, situación que suele ser muy diferente de un embalse a otro. Pérez (2000) para la presa El Salto, Sinaloa, México encontró dominancia de las hembras (1M:2H). Olague (2007) encontró que la proporción sexual de la lobina en la presa José López Portillo, Sinaloa, México fue a favor de las hembras en una relación $1.26 \mathrm{H}: 1 \mathrm{M}$. Un estudio sobre lobina realizado en el lago Bracciano, Italia se reporta una relación entre sexos de 0.8:1 a favor de las machos (Marinelli et al. 2007). Quizás la diferencia en la proporción de sexos en las poblaciones muestreadas de $M$. salmoides en los diferentes embalses tenga que ver con su comportamiento reproductivo; es decir, que el macho se encarga de construir el nido y cuida los huevecillos hasta su eclosión por lo que su movimiento se restringe alrededor del nido. Por su parte la hembra, una vez que desova, emigra hacia zonas más profundas del embalse. Granado-Lorencio (2000), señala que la proporción sexual de estos peces cambia a través de la vida como resultado de diferencias en el estrés reproductivo tanto por los machos como las hembras. 
El proceso de maduración de los productos sexuales de M. salmoides en la presa Bacurato, señala que el desarrollo de sus gónadas se presenta desde septiembre, mes en que aparecen especímenes en fase inicial de maduración, la cual se prolonga hasta abril, aunque la actividad reproductiva más importante se presenta de enero a marzo. Durante este periodo de tiempo, de acuerdo con García-Sosa (2010), el embalse registra una temperatura del agua en superficie de $21.5^{\circ} \mathrm{C}$ en enero y $25.6^{\circ} \mathrm{C}$ en marzo. Rodríguez-Sánchez et al. (2009) en el lago Primera de Palos, España, señalan temperaturas entre 20 y $25^{\circ} \mathrm{C}$ como ambiente propicio para la reproducción de M. salmoides. En presas de la región como El Salto, Sinaloa, México, la fase IV (maduros) aparece desde febrero y tiene el porcentaje más alto en abril (Osuna 2003). En algunos embalses de México, particularmente en la presa Vicente Guerrero (Tamaulipas), la lobina negra se reproduce entre noviembre y febrero (Roque 1992). Torres (1988) encontró que en la presa Rodrigo Gómez, Nuevo León, México el periodo reproductivo de M. salmoides abarca de febrero a mayo. En cuerpos de agua de otras regiones del mundo tal como el lago Trasimeno (Umbria, Italia) M. salmoides se reproduce principalmente en mayo (Lorenzoni et al. 2002), en tanto que en el lago Bracciano (Centro de Italia), establecen la época de reproducción entre diciembre y febrero (Marinelli et al. 2007). En el lago Chicalma (Mozambique) la reproducción ocurre en agosto y septiembre (Weyl \& Hecht 1998). Dadzie \& Aloo (2008) reportan que esta especie se reproduce por un periodo de ocho meses que abarcan desde el mes de junio a enero, con máximos en agosto y noviembre en el lago Naivasha, Kenya. En todos los casos hay coincidencia en que es la temperatura del agua el principal factor ambiental que influye en la reproducción de la especie, lo cual ocurre cuando se registran valores entre 20 y $25^{\circ} \mathrm{C}$. Criscoulo (2005) establece que la reproducción de peces es un proceso multifactorial, en el que el medio ambiente juega un importante papel. La lobina en el embalse estudiado, al parecer se ve estimulada a iniciar su reproducción cuando la temperatura del agua baja alrededor de los $21^{\circ} \mathrm{C}$ (entre enero y marzo), época en la que es la más fría del año.

El índice gonadosomático (IGS) viene a ser un indicador de la madurez de los productos sexuales en la mayoría de los peces, ya que conforme las gónadas se van desarrollando el IGS se incrementa (Rodríguez 1992). Aunque desde diciembre se registra un incremento, los máximos valores de IGS se presentan de enero a marzo, confirmando lo encontrado en el seguimiento de los grados de madurez. El seguimiento de evolución de la madurez de los productos sexuales y el comportamiento en el tiempo del índice gonadosomático, permiten establecer que la especie objeto de estudio se reproduce una vez al año. Osuna (2003) reporta que el índice gonadosomático de esta especie en la presa El Salto, Sinaloa, México, registra los valores más altos de enero a marzo, señalando que el proceso reproductivo es continuo hasta mayo.

La talla de primera madurez para esta especie en esta represa se estableció en $33.7 \mathrm{~cm}$ de longitud total. Este dato resulta similar a lo reportado por Olague (2007) en la presa José López Portillo, Sinaloa, México con $32.6 \mathrm{~cm}$. Por su parte, Valdez (1974) encontró una hembra madura con una longitud total de $23 \mathrm{~cm}$ en la presa Vicente Guerrero muy similar a lo que encontraron Beltrán et al. (1993) en las presa El Salto, Sinaloa, México, pues mencionan que esta especie se reproduce a talla mayores a los $20 \mathrm{~cm}$ de longitud total y Beamish et al. (2005), señalan los machos de M. salmoides en el lago Manyame, Zimbabwe, África inicia su reproducción a una talla de $18 \mathrm{~cm}$ y $75 \mathrm{~g}$ de peso (alrededor de su primer año de vida) y a $23 \mathrm{~cm}$ y $125 \mathrm{~g}$ las hembras (en su segundo año de vida).

La condición general de los peces es una respuesta a los cambios fisiológicos provocado por desviaciones en la canalización de energía (ya sea por fines reproductivos y/o almacenamiento de sustancias de reservas) a cambios en respuesta al medio ambiente o disponibilidad de alimento (Rodríguez 1992). El factor de condición de $M$. salmoides en la presa Gustavo Díaz Ordaz en ambos sexos alcanza sus valores 
más altos en invierno, aunque en el caso de los machos disminuye después de diciembre y continua hasta mayo de manera más marcada que en las hembras, lo que indica que va asociado a la época de reproducción de la especie, ya que al madurar las gónadas se incrementa el peso total del espécimen. Beltrán \& Sánchez (1990), señalan que en el embalse los Horcones, el comportamiento del factor de condición en $M$. salmoides se asocia a la época de reproducción, ya que los valores más bajos se presentaron en abril y mayo, meses en los que la actividad reproductiva fue más intensa. Resultados parecidos reporta Roque (1992) quien describe que en la presa Vicente Guerrero, México, el periodo reproductivo de esta especie se presenta en noviembre y enero, lo cual se confirma mediante el factor de condición.

Es posible inferir el potencial productivo de los peces por medio de la fecundidad (Begenal 1978). En la presa Gustavo Días Ordaz se encontró que $M$. salmoides ovipositó un promedio de $32294 \pm 12878.7$. Elizondo-Garza (1996) reportó para esta especie una fecundidad promedio de 14911 en la presa Vicente Guerrero, México. Por su parte, Torres (1988) encontró que una hembra de $29.4 \mathrm{~cm}$ y $346 \mathrm{~g}$ ovipositó 6856 ovocitos, y otra de $43.2 \mathrm{~cm}$ y 1306g 163881 ovocitos, en la presa Rodrigo Gómez, México. Como conclusión, Davis \& Lock (1997) señalaron que aunque una hembra oviposite 80000 ovocitos, el promedio oscila alrededor de 4000 por libra (553.6g) de pez. En embalses de la Península Ibérica, se reportó una fecundidad para M. salmoides de 10000 ovocitos (Doadrio 2001), y de acuerdo con Rodríguez-Sánchez et al. (2009) en el lago Primera de Palos, España, la máxima fecundidad que se encontró fue de 16850 ovocitos en una hembra de $44 \mathrm{~cm}$.

Contrario a lo encontrado por la mayoría de los estudios de fecundidad de la lobina negra en cuanto a que es una especie muy prolífica, Dadzie \& Aloo (2008) en el lago Naivasha, Kenia, encontraron que en especímenes de talla entre $25.9-29.9 \mathrm{~cm}$ y $371-550 \mathrm{~g}$ de peso, contaban con un promedio de 2203 ovocitos, valores muy por debajo de lo reportado por otros estudios. Zaniboni-Filho \& Nuner (2004) reportan que la calidad y la cantidad del alimento del reproductor puede generar problemas reproductivos al inducir la reabsorción de ovocitos vitelogénicos, afectando la vitelogénesis, y generando por lo tanto menor número de ovocitos maduros influyendo directamente sobre la fertilidad.

Por la importancia que este recurso representa para la comunidad de pescadores y el mantenimiento de la pesca deportiva-recreativa en el embalse, con el fin de su conservación basada en un buen manejo del recurso pesquero, se recomienda no afectar su proceso de reproducción al evitar su captura durante los meses de enero a marzo, y no capturar organismos menores a $33 \mathrm{~cm}$ ni mayores de 40 , por ser juveniles en el primer caso y por ser especímenes que atraen al turismo nacional y extranjero en el segundo.

\section{AGRADECIMIENTOS}

Expresamos nuestro agradecimiento a los revisores anónimos del manuscrito, a Dominico Voltolina L. y a la Federación de Cooperativas de la presa Gustavo Díaz Ordaz "Bacurato" por todas las facilidades prestadas para llevar a cabo esta investigación.

\section{RESUMEN}

Micropterus salmoides es un pez de importancia para la pesca deportiva en la mayoría de los embalses de México y del mundo, por lo que en el embalse Gustavo Díaz Ordaz se realizó un estudio sobre aspectos reproductivos entre agosto 2008 y marzo 2011. Se recolectaron 938 organismos, a los cuales se les midió la longitud ( $\mathrm{Lt} \mathrm{cm}$ ), peso ( $\mathrm{Pt}$ g), se determinó el sexo, índice gonadosomático, factor de condición, fecundidad y talla de primera madurez. La longitud y peso de los ejemplares recolectados varió de 15.9 a $63 \mathrm{~cm}(37.4 \pm 78.0)$ y de 57 a $4431 \mathrm{~g}(731.7 \pm 619.0)$, respectivamente. La relación peso total-longitud total se ajustó a un modelo tipo potencial, el crecimiento fue alométrico positivo, no se obtuvieron diferencias significativas entre machos y hembras $(\mathrm{F}=0.9955, \mathrm{p}=0.3187)$. La proporción macho-hembra fue 1:0.83. Los desoves masivos inician en diciembre y finalizan en abril. La talla de primera reproducción fue de $33.7 \mathrm{~cm}$ y la fecundidad promedio de $32294 \pm 12878.7$ ovocitos/hembra. El índice gonadosomático se mantuvo bajo desde mayo hasta noviembre y 
alto entre enero y marzo. El factor de condición registró valores altos previos al desove y disminuyó al final del periodo reproductivo.

\section{REFERENCIAS}

Anderson, R.O. 1980. Proportional Stock Density (PSD) and Relative Weight (Wr): Interpretive indices for fish population and communities, p. 27-33. In S. Gloss \& B. Shupp (eds.). Practical fisheries management: more with less in the 1980s. Proceedings of the American Fisheries Society, Ithaca, Nuava York, EE.UU.

Anuario estadístico de pesca. 2009. Estadísticas pesqueras. Comisión Nacional de Acuacultura y Pesca, México D.F., México.

Bagenal, B.T. 1978. Aspects of fish fecundity, p. 75-101. In S.D. Gerring (ed.). Ecology for freshwater fish production. Oxford, Londres, Reino Unido.

Beamish, C.A., A.J. Booth \& N. Deacon. 2005. Age, growth and reproduction of largemouth bass, Micropterus salmoides, in lake Manyame, Zimbabwa. Afr. Zool. 40: 63-69.

Beltrán, A.R. \& J. Sánchez. 1990. Estudio biológico y pesquero de la presa la Higueras, Sinaloa, México. Informe técnico, Universidad Autónoma de SinaloaSEP, México.

Beltrán, A.R., J. Sánchez \& G. Arroyo. 1993. Informe final del proyecto "Estudio Hidrológico y BiológicoPesquero de la presa El Salto, Sinaloa, México". Universidad Autónoma de Sinaloa-SEP, México.

Beltrán, A.R., J. Sánchez \& L.J.P. Ramírez. 2004. Plan de manejo de la presa Gustavo Díaz Ordaz, Sin. México. Informe técnico. Universidad Autónoma de Sinaloa, Mazatlán, México.

Castañeda, R.C. \& J.R. Silva. 2005. Dieta de la lobina en el centro de educación ambiental e investigación "Los Alamitos". Instituto del medio ambiente del estado de Aguas Calientes, UAAC, México.

Carlander, K. 1977. Handbook of Freshwater Fishery Biology. Ames: Iowa State University, Iowa, EE.UU.

Copp, G.H., M. Fox, F.N. Przybylski, F.N. Godinho \& A. Vila-Gispert. 2004. Life-time growth patterns of pumpkinseed Lepomis gibbosus introduced to Europe, relative to native North American populations. Fol. Zool. 53: 237-254

Criscuolo, U.E. 2005. Bases fisiológicas de la reproducción de los peces en el trópico, p 23-42. In P.D. Victoria, P.M.A. Landines \& O.A.I. Sanabria (eds.). Reproducción de los peces del trópico. INCODER, Colombia.

Dadzie, S. \& P.A. Aloo. 2008. Reproduction of the North American blackbass, Micropterus salmoides
(Lacépède), in an equatorial lake, Lake Naivasha, Kenya. Aquaculture Res. 21: 449-458.

Davis, J.T. \& J.T. Lock. 1997. Largemouth bass: biology and life history. Texas Cooperative Extension, Southern Regional Aquaculture Center, Publication 200, Stillwater, Oklahoma.

Doadrio, I. 2001. Atlas y libro rojo de los peces continentales de España. Dirección General de Conservación de la Naturaleza/Museo Nacional de Ciencias Naturales, Madrid.

Elizondo-Garza, R. 1996. Caracterización biológico-pesquera de la presa Vicente Guerrero (Las Adjuntas), Tamaulipas, México, con análisis de capturas de tilapia y lobina negra. INP, SEMARNAP. Ciencia Pesquera 13: 37-54.

García-Sosa, F. 2010. Comportamiento de la temperatura y su influencia en la distribución de algunos parámetros fisicoquímicos en el embalse Gustavo Díaz Ordaz, Sinaloa, México. Tesis de Licenciatura, Universidad Autónoma de Sinaloa, Sinaloa, México.

Godinho, F.N. \& M.T. Ferreira. 1996. The application of size-structure indices to Micropterus salmoides (Lacépède, 1802) and Lepomis gibbosus (L. 1758) populations as a management tool for southern Iberian reservoirs. Publicaciones Especiales. Instituto Español. Ocean. 21: 275-281.

Granado-Lorencio, C. 2000. Ecología de comunidades. El paradigma de los peces de agua dulce. Universidad de Sevilla, España.

Guzmán-Arroyo, M., J.I. Rojas \& F. Vera-Herrera. 1978. Crecimiento y aspectos poblacionales de la Lobina negra Micropterus salmoides Lacépède, en el lago de Camécuaro, Michoacán (Pisces: Centrachidae). Anal. Cent. Cien. Mar Limnol. UNAM. 6: 53-68.

Kesteven, G.L. 1975. Manual de ciencia pesquera. Organización de las Naciones para la Agricultura y la Alimentación (FAO), Roma, Italia.

Lorenzoni, M.M., A. Corboli, J.M. Dorr, G. Giovinazzo, S. Selvi \& M. Mearelli. 2002. Diets of Microptrerus salmoides lac. and Esox lucius L. in lake Trasimeno (Umbria, Italia) and their diet overlap. Bull. Fran. Pech. Piscic. 365/366: 537-547.

Marinelli, A., M. Scalici \& G. Gilbetini. 2007. Diet and Reproduction of Largemouth bass in a Recently Introduce Population, Lake Bracciano (Central Italy). BFPP/Bull. Fr. Pêche Piscic. 385: 53-68.

Medina, M., C. Pacheco \& R. Ruiz. 1994. Aspectos biológicos y de crecimiento de la lobina negra (Micropterus salmoides) Lacépède, en la presa El Salto, Elota, Sinaloa, México. Tesis de Licenciatura, Universidad Autónoma de Sinaloa. Sinaloa, México.

Nicola, G., A. Almodóvar \& B. Elvira. 1996. The diet of introduced largemouth bass, Micropterus salmoides, 
in the natural park of the Ruidera Lakes, Central Spain. Pol. Arch. Hydrob. 43: 179-184.

Olague, R.A. 2007. Aspectos poblacionales, reproductivos y alimenticios de la lobina negra Micropterus salmoides (Lacépède 1802) en la presa José López Portillo "El Comedero", Sinaola, México. Tesis de Licenciatura, Facultad de Ciencias del Mar, Universidad Autónoma de Sinaola. Sinaola, México.

Osuna, C.A.L. 2003. Comportamiento reproductivo de lobina negra (Micropterus salmoides Lacépède 1802) en la presa Aurelio "El Salto", Sinaloa, México. Tesis de Licenciatura, Universidad Autónoma de Sinaloa, Sinaloa, México.

Page, L. \& B. Burr. 1991. A Field Guide to Freshwater Fishes. Boston, Massachussetts, Houghton Mifflin, EE.UU.

Pérez, Q.M.R. 2000. Determinación de la edad y crecimiento de (Micropterus salmoides) en la presa Aurelio Benassini Vizcaíno "El Salto". Tesis de Licenciatura, Universidad Autónoma de Sinaloa, Sinaloa, México.

Ricker, W.E. 1975. Computation and interpretation of biological statistics of fish populations. Fish. Res. Board Can. Bull. 191: 1-332.

Robins, H.W. \& H.W.C. Grimmon. 1974. The black bass in America and overseas, Publications Division Biomanagement and Research Enterprise, Ontario, Canada.

Rodríguez, G.M. 1992. Técnicas de evaluación cuantitativa de la maduración gonádica en peces. A.G.T. Editor, México.

Rodríguez-Sánchez, V., A. Rodríguez-Ruiz \& R. SánchezCarmona. 2009. Largemouth bass, Micropterus salmoides, growth and reproduction in Primera de Palos' lake (Huelva, Spain). Fol. Zool. 58: 436-446.

Roque, V.I. 1992. Estudio Biológico Pesquero de la Lobina Negra (Micropterus salmoides), en la presa Vicente Guerrero (Las adjuntas), Tamaulipas. Tesis de Licenciatura, Universidad Nacional Autónoma de México, D.F., México.
Rosenblum, P.M., J. Pudney \& Y. Callard. 1987. Gonadal morphology enzyme histochemistry and plasma steroid level during annual reproductive cycle of male and female brown bullhead catfish, Ictalurus nebulosus Lesueur. J. Fish. Biol. 31: 325-341.

Saborido, R.F. 2005. Ecología de la reproducción y potencial reproductivo en poblaciones de peces marinos. Instituto de Investigaciones Marinas, Universidad de Vigo, España.

Schulz, U.H. \& M.E. Leal. 2005. Growth and mortality of black bass, Micropterus salmoides (Pisces, Centrarchidae, Lacépède, 1802) in a reservoir in southern Brazil. Braz. J. Biol. 65: 363-369.

Sparre, P. \& S. Venema. 1997. Introducción a la evaluación de recursos pesqueros tropicales. Parte I. Manual. FAO Documento Técnico de Pesca 306, Roma, Italia.

Torres, M.M. 1988. Madurez, ciclo gonádico y fecundidad de la lobina Micropterus salmoides como fundamento para determinar la veda reproductiva. Informe técnico, Comité Técnico Consultivo para la pesca deportiva en el estado de Nuevo León. Universidad Autónoma de Nuevo León, México.

Valdez, G.A. 1974. Biología pesquera de Micropterus salmoides (LACEPEDE), en la presa Marte R. Gómez, Tamaulipas. Tesis profesional, Universidad Autónoma de Nuevo León, Monterrey, México.

Weyl, O.L.F \& T. Hecht. 1998. A successful population of largemouth bass, Micropterus salmoides, in a subtropical lake in Mozambique. Environ. Biol. Fish. 54: 53-66.

Zaniboni-Filho, E. \& A.P. Nuner. 2004. Fisiologia da reproducao e propagacao artificial dos peixes, p. 45-73. In J.E.P. Cyrino, E.C. Urbinati, D.M. Fracalossi \& N. Castagnolli. (eds.). Topicos especiais em piscicultura de agua doce tropical intensiva, Sao Paulo, Brasil.

Zar, J.H. 1984. Biostatistical analysis. Prentice Hall International, Nueva Jersey, EE.UU. 
